\title{
Loss of Environmental Enrichment Increases Vulnerability to Cocaine Addiction
}

\author{
Joëlle Nader ${ }^{1,2,3,5}$, Chauvet Claudia ${ }^{2,3,5}$, Rana El Rawas ${ }^{2,3,5}$, Laure Favot ${ }^{3,4}$, Mohamed Jaber ${ }^{1,2,3}$, \\ Nathalie Thiriet* ${ }^{* 1,2,3,6}$ and Marcello Solinas ${ }^{1,2,3,6}$ \\ 'INSERM U-1084, Experimental and Clinical Neurosciences Laboratory, Neurobiology and Neuropharmacology Addiction Group, Poitiers, \\ France; ${ }^{2}$ Institut de Biologie et Physiologie Cellulaires, CNRS, Poitiers, France; ${ }^{3}$ University of Poitiers, I Rue Georges Bonnet, Poitiers, France; \\ ${ }^{4}$ Laboratoire Inflammation, Tissus Epithéliaux et Cytokines, Poitiers, France
}

\begin{abstract}
Life experiences, especially during critical periods of maturation, such as adolescence, can dramatically affect vulnerability to diseases at adulthood. Early exposure to positive environmental conditions such as environmental enrichment (EE) has been shown to reduce the occurrence and the intensity of neurological and psychiatric disorders including drug addiction. However, whether or not exposure to EE during early stages of life would protect from addiction when, at adulthood, individuals may find themselves in non-enriched conditions has not been investigated. Here we show that switching mice from EE to non-enriched standard environments not only results in the loss of the preventive effects of EE but also increases the rewarding effects of cocaine. This enhanced vulnerability is associated with emotional distress and with increased levels in the mRNA levels of corticotropin releasing factor (CRF) in the bed nucleus of the stria terminalis (BNST), as well as with increases in CREB phosphorylation in the BNST and in the shell of the nucleus accumbens. The increased sensitivity to the rewarding effects of cocaine is completely blocked by the CRF antagonist antalarmin, confirming a major role of the CRF system in the negative consequences of this environmental switch. These results indicate that positive life conditions during early stages of life, if they are not maintained at adulthood, may have negative emotional consequences and increase the risks to develop drug addiction.

Neuropsychopharmacology (2012) 37, I579-1587; doi:I0.1038/npp.20 I2.2; published online I5 February 2012
\end{abstract}

Keywords: addiction; emotion; adolescence; vulnerability factors; life experience

\section{INTRODUCTION}

Compelling evidence demonstrates that life experiences and environments, especially during early stages of life, highly influence the effects of drugs of abuse and have a major role in the development of drug addiction (Enoch, 2011; Swadi, 1999). In animal models of addiction, the importance of environmental conditions on subsequent effects of drugs of abuse has been clearly established. In particular, chronic exposure to stress induces neuronal adaptations that result in increased rewarding effects of drugs and higher vulnerability to addiction (Goeders, 2002; Koob, 2008; Kreek et al, 2005; Marinelli and Piazza, 2002; Sinha, 2001). The corticotropin releasing factor (CRF) system (Arborelius et al, 1999) and transcription factors, such as CREB (Carlezon et al, 2005),

\footnotetext{
*Correspondence: Dr N Thiriet, INSERM U- 1084, Experimental and Clinical Neurosciences Laboratory, Neurobiology and Neuropharmacology Addiction Group, Université de Poitiers, I Rue George Bonnet, 86022, Poitiers, France, Tel: 335493662 09, Fax: 335494540 I4, E-mail: nathalie.thiriet@univ-poitiers.fr

${ }^{5}$ These authors contributed equally to the work.

${ }^{6}$ These authors contributed equally to the work.

Received 7 August 2011; revised II December 201।; accepted 29 December 2011
}

appear to have a major role in stress-induced alterations of drugs' effects (Carlezon et al, 2005; Koob, 2008). For example, it has been recently shown that chronic stress increases the rewarding effects of cocaine by increasing CRF signaling and consequently pCREB levels in several brain regions involved in stress reactions and reward processes (Kreibich et al, 2009), and CRF can increase phosphorylation of CREB in striatal neurons (Stern et al, 2011).

In parallel with these findings, accumulating evidence indicates that exposure to environmental enrichment (EE) during early stages of life reduces the effects of drugs of abuse and decreases the vulnerability to develop addiction (Carroll et al, 2009; Laviola et al, 2008; Solinas et al, 2010; Stairs and Bardo, 2009). For example, mice reared in EE from weaning to adulthood show reduced conditioned place preference (CPP) to cocaine and heroin (El Rawas et al, 2009; Solinas et al, 2009) but not to methamphetamine (Thiriet et al, 2011). In addition, rats reared in EE show reduced self-administration of amphetamine and cocaine (Bardo et al, 2001; Green et al, 2009; Green et al, 2002; Stairs et al, 2006). Although in most experiments investigating the effects of EE on drug addiction, animals were constantly exposed to $\mathrm{EE}$, an important question that needs to be addressed is whether exposure to EE during early stages of 
life would protect from addiction even if enrichment were discontinued.

In this study, we reared mice from weaning to adulthood in either a standard environment (SE) or EE, we then switched them to EE or SE and after 7, 30, or 90 days, we investigated the ability of cocaine $(10 \mathrm{mg} / \mathrm{kg})$ to induce CPP. Surprisingly, we found that, when an environmental switch occurred, exposure to EE during early stages of life not only did not protect from cocaine but also made mice more vulnerable to its reinforcing effects. This effect was agedependent because mice that experienced EE and switch from EE to SE at adulthood, developed CPP similarly to control groups. The switch from EE to SE in young mice was associated with a negative emotional state and with increases in CRF levels in the bed nucleus of the stria terminalis (BNST) and increased phospo-CREB levels in the BNST and the nucleus accumbens (NAc) shell. Finally, we demonstrated that chronic blockade of $\mathrm{CRF}_{1}$ receptors $\left(\mathrm{CRF}_{1} \mathrm{Rs}\right)$ during the 7 days, intervening between the environmental switch and CPP conditioning, completely prevented switch-induced enhancement of the rewarding effects of cocaine.

\section{MATERIALS AND METHODS}

\section{Subjects}

Male C57Bl/6J mice (Janvier, Le Genest Saint Isle, France) were housed in a temperature-controlled environment on a 12-h light/dark cycle with the lights on from 0700-1900 hours and had ad libitum access to food and water. All experimentation was conducted during the light period. Experiments were carried out in accordance with the European Communities Council Directive of 24 November 1986 (86/609/EEC) for the care of laboratory animals.

\section{Housing Environmental Conditions}

After weaning ( 3 weeks of age), mice were randomly divided in two different housing environmental conditions, SE or EE, and kept in these housing conditions for 2 months. In rodents, this period encompasses adolescence and young adulthood. SE consisted of common cage housing $\left(25 \times 20 \times 15 \mathrm{~cm}^{3}\right)$. The EE consisted of larger $(60 \times 38 \times$ $20 \mathrm{~cm}^{3}$ ) cages containing constantly a small house and a running wheel for voluntary exercise, and four to five toys that were changed once a week with new toys of different shape and color. All mice were housed in groups of 4 .

A separate group of animals was housed in SE until adulthood (approximately 11 weeks of age). Subsequently, mice were randomly divided in two different housing environmental conditions, SE or EE, and kept in these housing conditions for 2 months and environmental switch was performed only thereafter (adult control group).

\section{General Experimental Design}

At adulthood (11 weeks of age), half of the mice in one environmental condition were switched to the other condition and kept in the animal facility until testing or killing (Figure 1). CPP experiments were conducted 7, 30, or 90 days after the switch. This allowed selecting a 7-day time point for all other procedures. Control groups of animals were kept in their original environment for equivalent periods of time. We compared four experimental groups: (1) SE-SE mice that were housed in SE throughout the experiment; (2) SE-EE mice that were housed in SE until adulthood and then switched to EE; (3) EE-EE mice that were housed in EE throughout the experiment and (4) EESE mice that were housed in EE until adulthood and then switched to SE. For adult control experiments, we obtained the same four experimental groups, and tests for CPP were performed 7 days after the switch.

For antalarmin experiments, a different $2 \times 2$ design was used with two environmental conditions (EE-SE and SE$\mathrm{SE}$ ) and two treatments (antalarmin or vehicle) (Figure 5a). Starting immediately after the switch, mice were injected twice per day (1800-0800 hours) with the $\mathrm{CRF}_{1} \mathrm{R}$ antagonist antalarmin (Sigma-Aldrich; www.sigmaaldrich.com) at a dose of $10 \mathrm{mg} / \mathrm{kg}$. A total of 13 injections were administered with the last administration being 15-18 hours before the preconditioning session of CPP in order to avoid any interference of antalarmin treatment and CPP procedures.

\section{Rewarding Effects of Cocaine}

CPP experiments with cocaine $(10 \mathrm{mg} / \mathrm{kg}$ i.p. $)$ were performed as previously described (Solinas et al, 2009). CPP procedure consisted of three phases: preconditioning (one 30-min session), conditioning (two 30-min sessions per day for 3 days), and test (one 30-min session). Preference scores, which served as a measure of rewarding effects of

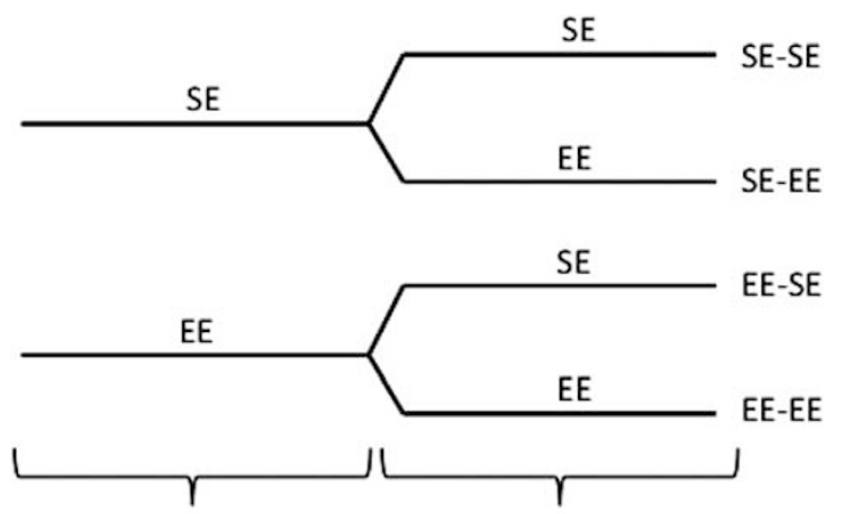

Pre-Adolescence, Adolescence and

Adulthood young adulthood
60 days
7,30 or 90 days

Figure I Schematic representation of the experimental design. Mice were initially housed either in SEs or EEs from weaning to adulthood. At adulthood (approximately post-natal day, PND 77), mice were either switched to the other environmental condition or they were kept in the same environmental condition as controls. CPP experiments were conducted 7, 30, or 90 days after the switch. Note that different groups of mice were used for each time point. Note also that for the adult group, a similar protocol was used, but exposure to EE started at adulthood (approximately PND 77) and environmental switch occurred after 8 weeks (approximately PND 135). 
cocaine, were calculated by subtracting the time in seconds spent during the pretest from the time spent during the test day in the compartment paired to cocaine injections.

\section{Emotional Distress}

In separate groups of mice, open field (OF), forced swim test (FST), and splash test were performed to measure emotional distress. For the OF test, animals were put in the middle of a white arena $\left(50 \times 50 \times 50 \mathrm{~cm}^{3}\right)$ and left free to explore it for $10 \mathrm{~min}$. The behavior of mice was recorded using a video camera and analyzed by Viewpoint software (Viewpoint Life Sciences, Lyon, France; www.vplsi.com/ news.php). A circle of $40 \mathrm{~cm}$ diameter was virtually drawn in the center of the arena and the percentage of time spent in this area over the total time was used as a primary measure of anxiety-like behavior. For FST experiments, mice were placed in a beaker $\left(21.4 \times 17.2 \mathrm{~cm}^{2}\right)$ of water $(23-$ $25^{\circ} \mathrm{C}$ ) and their behavior was recorded using a video camera for $6 \mathrm{~min}$ and scored by an observer blind to the environmental manipulation. Immobility time, defined as the absence of all movements except those required to keep the head of the mouse above the water, was measured. The splash test was used to measure emotional distress (Santarelli et al, 2003; Surget et al, 2008). In brief, the dorsal coat of the mouse was sprinkled with a $10 \%$ sucrose solution with a manual sprayer (two pulverizations delivering approximately $1 \mathrm{ml}$ ). Because of its viscosity, the sucrose solution triggers a licking and grooming behavior. The first grooming latency and the time spent grooming were recorded for a period of $5 \mathrm{~min}$.

\section{Quantitative Real Time Reverse Transcription-PCR (RT-PCR)}

Seven days after the environmental switch, mice were decapitated and brains were removed, immediately frozen on dry ice, and stored at $-80^{\circ} \mathrm{C}$ until use. Sections $(200 \mu \mathrm{m})$ were cut using a cryostat and BNST or amygdala were punched out using a $1-\mathrm{mm}$ microdissecting needle. RNAs for RT-PCR quantification were obtained as previously described (Thiriet et al, 2008). The sequences of primers used were designed using Primer-BLAST software (http:// www.ncbi.nlm.nih.gov/tools/primer-blast/index.cgi?LINK $\mathrm{LOC}=$ BlastHome). The sequence for $\mathrm{CRF}_{1} \mathrm{R}$ was the same used by Sztainberg et al (2010). Samples were normalized to control housekeeping genes (GAPDH) and reported according to the delta CT method as $2^{-\Delta \mathrm{CT}}=2^{-(\mathrm{CT}}$ gene of interest-CT GAPDH), where CT corresponds to the cycle threshold when fluorescence is first detected. The changes in $2^{-\Delta \mathrm{CT}}$ were normalized over SE-SE values and used for analysis.

\section{Phospho-CREB Immunohistochemistry}

Seven days after the environmental switch, mice were anesthetized, transcardially perfused, and brain processed as previously described (Solinas et al, 2008). Antibodies directed at the phosphorylated form of CREB (Ser 133) (Cell signaling; www.cellsignal.com) were used. Pictures of sections (three per mouse) were taken using computerized image analysis software (MorphoStrider, ExploraNova, La Rochelle, France) and were quantified bilaterally using cell count analysis software (Mercator, ExploraNova, La Rochelle, France) by an experimenter blind to group assignments.

\section{Drugs}

Cocaine $\mathrm{HCl}$ was obtained from the Research Triangle Institute and dissolved in sterile saline solutions (0.9\%). Antalarmin (Sigma-Aldrich) was dissolved in 10\% DMSO in saline solutions. All drugs were administered i.p. in a volume of $1 \mathrm{ml} / 100 \mathrm{~g}$.

\section{Statistical Analysis}

All results are presented as group means ( \pm SEM). Differences between groups were assessed by one-way with environmental history as factor or two-way ANOVA with environmental history and time as factors. Results showing significant overall changes were subjected to StudentNewman-Keuls post-hoc test. Differences were considered significant when $P<0.05$.

\section{RESULTS}

\section{Switch from EE to SE Increases the Rewarding Effects of Cocaine}

Consistent with our previous findings (Solinas et al, 2009), cocaine induced significant preference in mice that were housed continuously in SE (SE-SE) but not in mice that were housed continuously in EE (EE-EE) (Figures 2a-c). In mice that were housed in SE from weaning to adulthood and were switched to EE before the beginning of CPP conditioning (SE-EE), cocaine still induced significant preference 7 days after the switch (Figure 2a). However, 30 and 90 days after the switch, SE-EE mice appear little or not at all sensitive to the rewarding effects of cocaine (Figures $2 \mathrm{~b}$ and c), indicating that extensive exposure to EE can have protective effects against addiction even when provided at adulthood. Surprisingly, and opposite to our predictions, in mice that were housed in EE from weaning to adulthood and were switched to SE (EE-SE), cocaine induced a very strong preference (500 s, ie, 200\% more than SE-SE controls) when CPP conditioning was performed 7 and 30 days after the environmental switch (Figures $2 \mathrm{a}$ and $\mathrm{b}$ ). Only after 90 days in SE, EE-SE mice returned to preference scores comparable to controls (SE-SE) (Figure 2c). The effects of environmental switch as a function of time for each group are shown in Supplementary Figure 1. Statistical analysis revealed a significant effect of environmental history $\left(\mathrm{F}_{3,119}=8.00, P<0.0001\right)$, time $\left(\mathrm{F}_{2,119}=6.80, P<0.01\right)$, and a significant environmental history $\times$ time interaction effect $\left(\mathrm{F}_{6,119}=3.36, P<0.01\right)$.

Subsequently, we investigated whether the effects of environmental switch depended on the age at which animals were exposed to EE before being switched or not to SE. When mice were housed in $\mathrm{EE}$ at adulthood, exposure to $\mathrm{EE}$ for 2 months (EE-EE), but not for 1 week (SE-EE), was able to block the development of cocaine CPP (Figure 2d). On the other hand, mice housed in EE at adulthood and then switched to SE for 1 week (EE-SE) showed a CPP that was similar to that of control SE-SE mice (Figure 2d). These results show that although the protective effects of $\mathrm{EE}$ are 


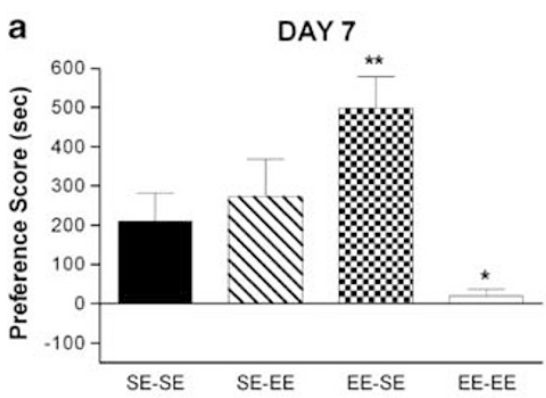

b
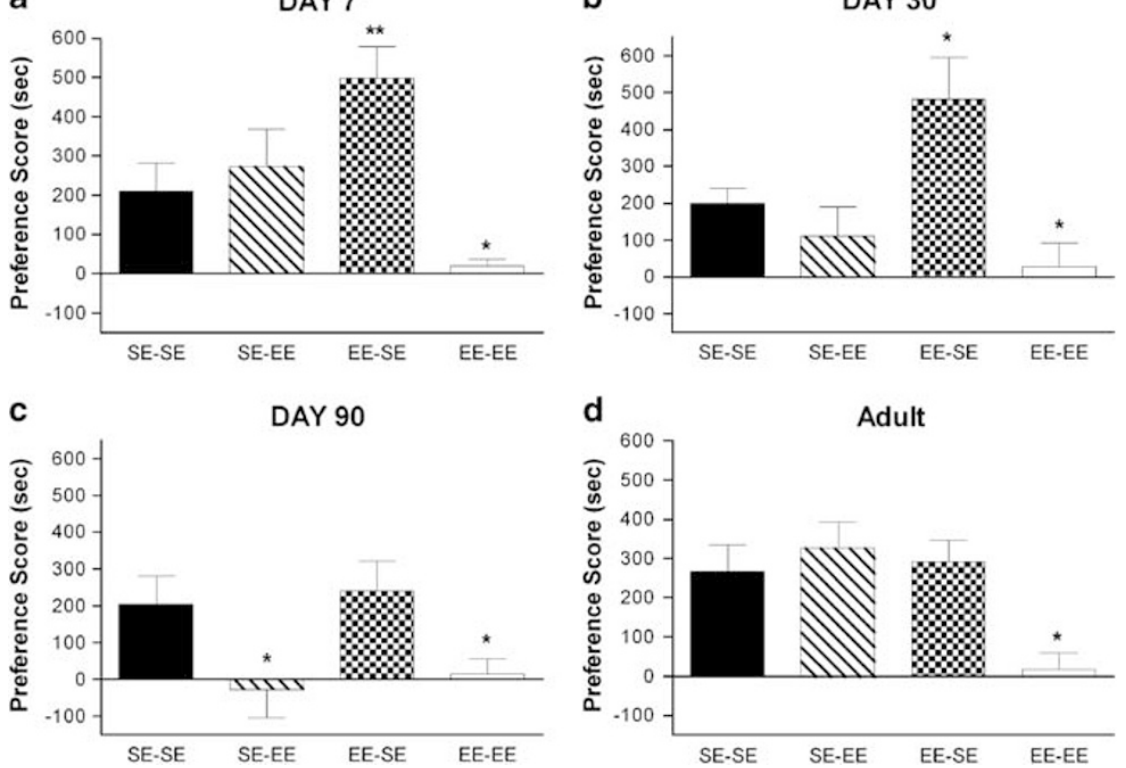

Figure 2 Effects of environmental switch on the rewarding effects of cocaine. In mice housed in EE or SE from weaning to adulthood, cocaine-induced CPP was investigated (a) 7, (b) 30, or (c) 90 days after the environmental shift in mice switched from SE to EE (SE-EE) or EE to SE (EE-SE) and their nonswitched controls (SE-SE and EE-EE). In mice housed in EE or SE for 2 months starting at adulthood (d), cocaine-induced CPP was investigated 7 days after the environmental shift. Preference score is the difference in the time $( \pm S E M)$ spent in the cocaine-paired compartment before and after conditioning $(n=10-1 \mid$ per group). Post Student-Newman-Keuls $* P<0.05$ and $* * P<0.01$ different from SE-SE controls.

rapidly lost after return to $\mathrm{SE}$, this switch at adulthood does not cause an increase in the rewarding effects of cocaine. Statistical analysis showed a significant effect of environmental history with EE differing from all other groups (oneway ANOVA, $\mathrm{F}_{3,36}=6.44, P<0.01$ ).

\section{Switch from EE to SE Produces a Negative Emotional State}

We then investigated whether the enhanced sensitivity to the rewarding effects of cocaine was associated with a negative emotional state. Seven days after the switch, in an OF test, mice switched from EE to SE showed low levels of exploration of the center of the arena, a behavior that is consistent with high levels of anxiety. These levels were comparable to those of SE-SE but significantly lower than those of both SE-EE and EE-EE mice (one-way ANOVA, environmental history, $\left.\mathrm{F}_{3,31}=4.94, P<0.01\right)$. On the other hand, in a FST procedure, we found that over a 6-min test EE-EE mice showed less immobility than other groups (Figure 3a) (one-way ANOVA, $\mathrm{F}_{3}, 33=3.35, \quad P<0.05$ ), confirming an antidepressant-like effect of enrichment (Laviola et al, 2008; Nithianantharajah and Hannan, 2009; Solinas et al, 2010). EE-SE mice did not differ from SE-SE or SE-EE mice in the total time of immobility during the 6min test (Figure 3b). However, a closer look at time courses revealed that EE-SE showed low immobility levels similar to EE-EE in the first 2-min bin of the test, but in the second and third 2-min bins, they showed immobility levels similar to those of SE-SE and SE-EE mice (Figure 3c). Indeed, when expressed as \% of initial immobility levels, EE-SE showed increases in immobility that were significantly higher than all other groups (Figure 3d) (one-way ANOVA, environmental history, $\left.\mathrm{F}_{3,33}=3.10, P<0.05\right)$. We interpreted low levels of immobility during the initial phase of the test as a consequence of motor fitness of EE-SE mice, due to months of physical activity in EE (Madronal et al, 2010; Munn et al, 2011; Pietropaolo et al, 2006). On the other hand, we interpreted the rapid increase in immobility found over the course of the FST session in EE-SE as a manifestation of enhanced behavioral despair and of an underlying negative emotional state. To confirm this hypothesis, we used the splash test, a procedure sensitive to chronic stress (Santarelli et al, 2003; Surget et al, 2008) that is independent from motor performance. We found that mice switched from EE to SE show significantly longer latency to start grooming behavior and spend less time grooming than all other groups of mice (Figure 3e) (one-way ANOVA, latency: $F_{3,40}=3.37, P<0.05$; grooming time: $F_{3,40}=5.12$, $P<0.01)$ confirming a low emotional state in these mice.

\section{Switch from EE to SE is Associated with Increases in mRNA Levels of CRF}

CRF is one of the major neurotransmitters involved in stress responses and emotions (Arborelius et al, 1999), and it has been recently demonstrated that the anxiolytic effects of EE depend on the CRF system in amygdaloid regions (Sztainberg et al, 2010). Therefore, we hypothesized that the emotional distress induced by the switch from EE to SE could be mediated by alteration in the expression of members of the CRF system in amygdaloid nuclei. Using quantitative RT-PCR, we found that switch from EE to SE is associated with a selective increase in CRF $\mathrm{mRNA}$ in the BNST (Figure 4a) (one-way ANOVA, $\mathrm{F}_{3,34}=3.10, P<0.05$ ) but not in the amydgala (Figure $4 \mathrm{~b}$ ). No significant changes in $\mathrm{CRF}_{1} \mathrm{R}$ or $\mathrm{CRF}_{2} \mathrm{R}$ mRNA were found in either the BNST or the amydgala (Figures $4 \mathrm{a}$ and $\mathrm{b}$ ). 


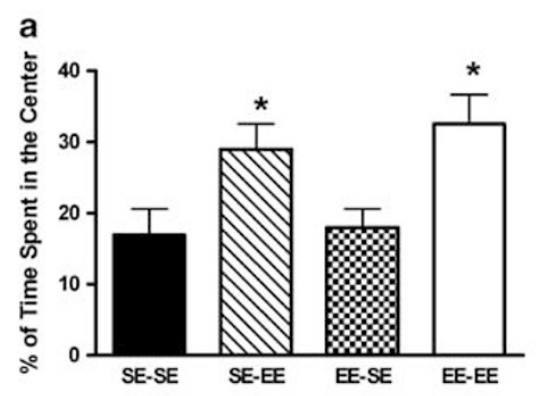

b
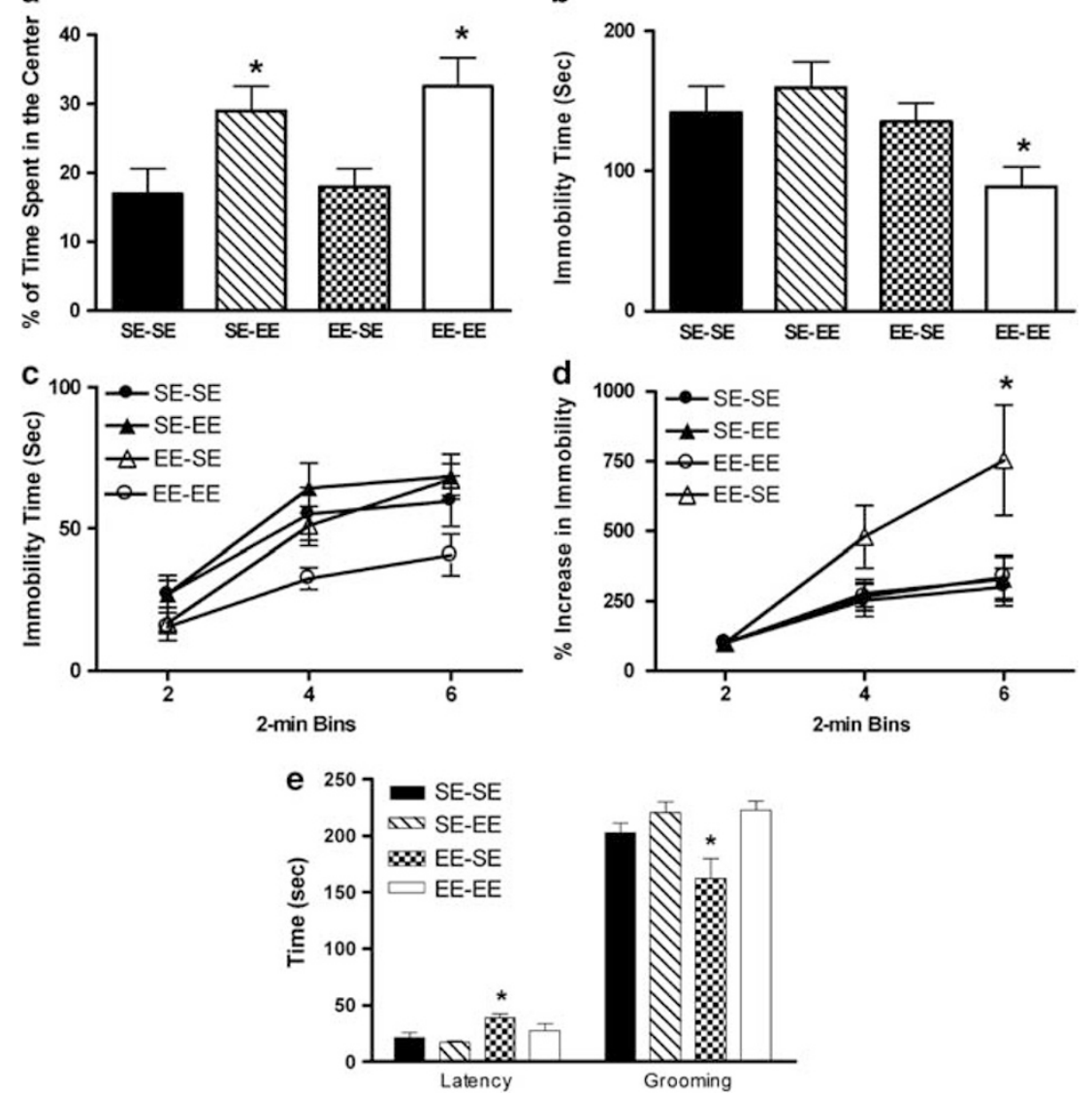

Figure 3 Effects of environmental switch on emotional responses. Stress responses measured in the OF, FST and splash test in mice switched from SE to EE (SE-EE) or EE to SE (EE-SE) and their non-switched controls (SE-SE and EE-EE) 7 days after the environmental switch. Panel (a) shows percentage of time spent in the center of the OF arena. Panel (b) shows total immobility and panel (c) shows time course for immobility during a 6-min FST. Panel (d) shows the percentage increase in immobility in the second and third 2-min FST compared with the initial 2-min bin. Panel (e) shows latency to grooming and time spent grooming in the splash test. Data are expressed as mean ( \pm SEM) of group values $(n=10-1 \mid$ per group). Post Student-Newman-Keuls $* P<0.05$ different from SE-SE controls.

\section{Blockade of $\mathrm{CRF}_{1} \mathrm{R}$ Eliminates the Enhanced Sensitivity to Cocaine Induced by Environmental Switch}

We then investigated whether the increased CRF tone associated with switch from EE to SE was responsible for the enhanced sensitivity to the rewarding effects of cocaine. For this, we switched animals and treated them with the $C_{R F} R$ antagonist antalarmin ( $10 \mathrm{mg} / \mathrm{kg}$ twice per day) or vehicle during the 7 days, occurring between the environmental switch and the beginning of cocaine CPP conditioning (Figure 5a). In order to verify that chronic treatment with antalarmin itself did not disrupt subsequent CPP, control mice were housed in SE and received antalarmin or vehicle according to the same protocol. We found that antalarmin completely abolished the increase in cocaine rewarding effects induced by switch from $\mathrm{EE}$ to $\mathrm{SE}$ (Figure $5 \mathrm{~b}$ ) (one-way ANOVA, $\mathrm{F}_{3,39}=3.32, P<0.05$ ).

Switch from EE to SE is Associated with Increases in pCREB Immunoreactivity Levels in the BNST and in the NAc Shell

It has been shown that repeated stress increases reactivity to cocaine through an increase in CREB phosphorylation in limbic areas and that these effects of stress are lost in mice lacking CREB (Kreibich et al, 2009). We therefore investigated whether environmental switch could lead to increases in levels of PCREB in several brain areas such as the NAc shell and core, the lateral septum (LS), the BNST, the basolateral amygdala (BLA), basomedial amygdala, and central amygdala (CeA) (Supplementary Figure 2). We found that switch from EE to SE increased pCREB immunoreactivity levels selectively in the BNST and NAc shell (Figure 6) (one-way ANOVA, NAc: $F_{3,26}=3.42, P<0.05$; BNST: $\left.\mathrm{F}_{3,26}=5.13, P<0.01\right)$ but not in the other regions.

\section{DISCUSSION}

Exposure to EE has been shown to have beneficial effects on neurodegenerative disorders, such as Parkinson Disease, Alzheimer Disease, and Huntington Disease, and psychiatric disorders such as anxiety, depression and schizophrenia (Baroncelli et al, 2010; Cirulli et al, 2010; Laviola et al, 2008; Nithianantharajah and Hannan, 2009; Rosenzweig and Bennett, 1996). In addition, $\mathrm{EE}$ has been shown to have both preventive and restorative effects on drug addiction (Carroll et al, 2009; Laviola et al, 2008; Solinas et al, 2010; Stairs and Bardo, 2009). For example, it has been recently reported that mice housed in EE from weaning to adulthood 
a

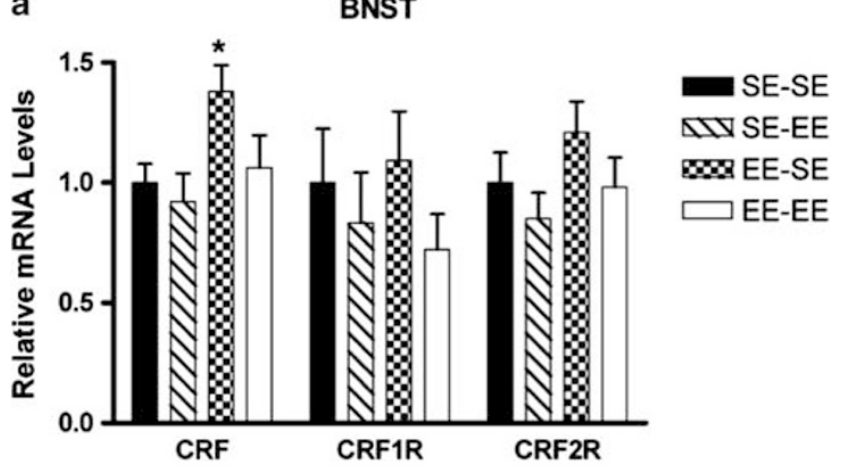

b

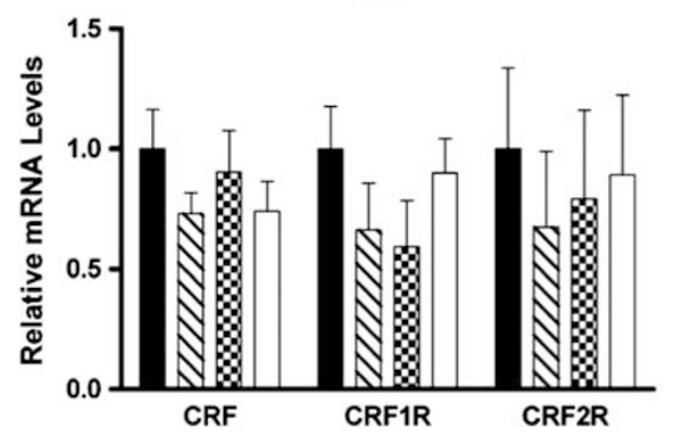

a

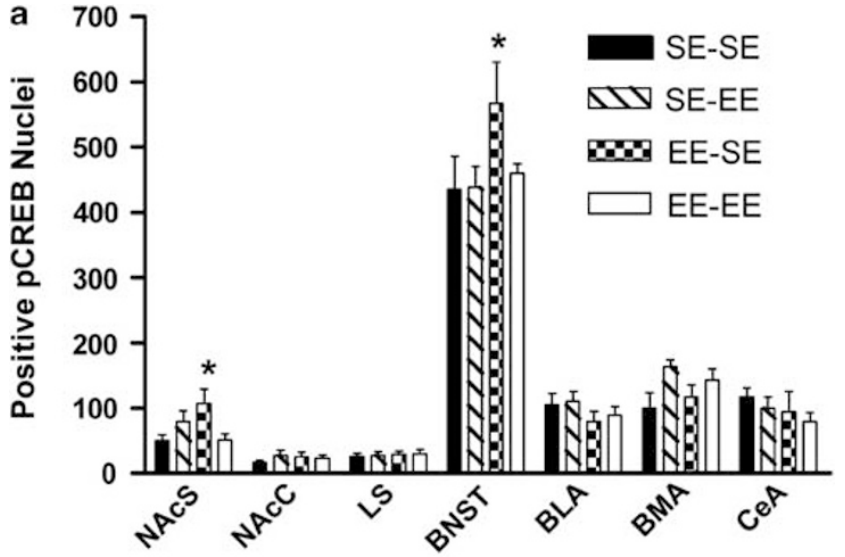

SE-SE

$\triangle I V S E-E E$ Q 20 EE-SE EE-EE

Figure 4 Effects of environmental switch on the mRNA expression of $C R F, C R F_{1} R$ and $C R F_{2} R$ in amygdaloid nuclei. mRNA levels of $C R F, C R F_{1} R$ and $\mathrm{CRF}_{2} \mathrm{R}$ in the (a) BNST and (b) amygdala in mice switched from SE to EE (SE-EE) or EE to SE (EE-SE) and their non-switched controls (SE-SE and $\mathrm{EE}-\mathrm{EE}) 7$ days after the environmental switch. A significant increase in the levels of CRF mRNA was found in the BNST only in EE-SE mice. Data are expressed as mean $( \pm$ SEM) of group values $(n=9-10$ per group). Post Student-Newman-Keuls $* P<0.05$ different from SE-SE controls.
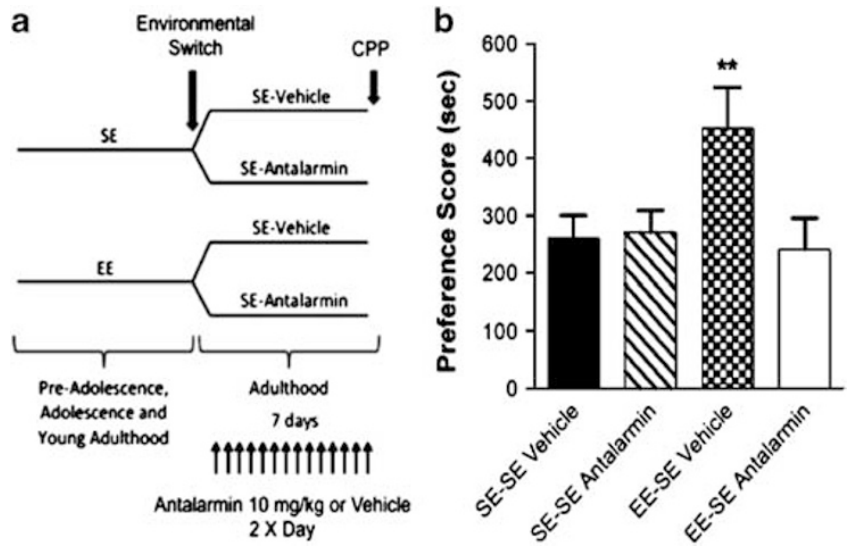

Figure 5 Effects of $C R F_{1} R$ blockade following environmental switch on cocaine-induced CPP. (a) Schematic representation of the experimental design. Cocaine-induced CPP was investigated in mice switched from EE to $\mathrm{SE}$ (EE-SE) and treated with the CRF, R antagonist antalarmin ( $10 \mathrm{mg} / \mathrm{kg}$ i.p. twice per day) or vehicle during the 7 days occurring between the switch and the beginning of the CPP conditioning sessions. Control mice were housed always in SE and were treated with antalarmin or vehicle. (b) Chronic treatment with antalarmin completely blocked the enhanced response to cocaine induced by the switch from EE to SE. Preference score is the difference in the time $( \pm S E M)$ spent in the cocaine-paired compartment before and after conditioning ( $n=|0-1|$ per group). Post Student-Newman-Keuls $* * *<0.01$ different from SE-SE controls.



Figure 6 Effects of environmental switch on pCREB levels. (a) pCREBpositive nuclei were quantified in the NAc shell, NAc core, the lateral septum (LS), the BNST, the basolateral amygdala (BLA), the basomedial amygdala (BMA), and the central amygdala (CeA) of mice switched from SE to $\mathrm{EE}$ (SE-EE) or EE to SE (EE-SE) and their non-switched controls (SE-SE and EE-EE) 7 days after the environmental switch. EE-EE mice show a significant increase in PCREB expression only in the NAc shell and the BNST. Data are expressed as mean ( \pm SEM) of group values $(n=7-8$ per group). Post Student-Newman-Keuls $* P<0.05$ different from SE-SE controls. (b) Representative photomicrograph illustrating quantitative data of pCREB-immunoreactive nuclei within the BNST for each group (scale bar, $10 \mu \mathrm{m})$.

show reduced rewarding effects to cocaine (Solinas et al, 2009), heroin, and morphine (El Rawas et al, 2009; Xu et al, 2007). Here we aimed at determining whether the positive effects of EE on cocaine vulnerability would persist after the discontinuation of EE. Surprisingly, the main finding of the present study is that discontinuation of EE leads to increases in the rewarding effects of cocaine in comparison with mice that have been exposed to neutral SE throughout the experiment. Thus, not only the positive effects of EE are not long lasting but also depriving mice from EE increases vulnerability to cocaine. Therefore, mice that have been exposed to a rich, stimulating environment could find a SE rather poor, and thus the switch from EE to SE would be perceived as stressful and aversive. Somewhat similarly, it has been shown that DBA mice that have been socially housed for 19 days and then switched to single housing selfadminister more cocaine than mice that are always kept in single housing (van der Veen et al, 2007). 
Given these somewhat unexpected results, we hypothesized that the switch-induced increase in cocaine rewarding effects was a consequence of a negative emotional stress. In fact, stress is known to increase the reinforcing effects of drugs (Goeders, 2002; Koob, 2008; Kreek et al, 2005; Marinelli and Piazza, 2002; Sinha, 2001), and accumulating evidence shows that exposure to stress increases the ability of cocaine to induce CPP (Kreibich et al, 2009; McLaughlin et al, 2006; McLaughlin et al, 2003). Consistent with this hypothesis, we found that emotional responses were altered in mice switched from EE to SE. In a FST, a measure of emotional behavior that is dependent on physical activity, we found that EE-SE mice initially show low levels of immobility probably because of motor fitness produced by months of physical activity in EE (Madronal et al, 2010; Munn et al, 2011; Pietropaolo et al, 2006). However, this apparent beneficial effect quickly disappeared and EE-SE mice showed increased immobility levels that were significantly higher than other groups, a behavior that we interpreted as a despair-like behavior. We confirmed low emotional responses in these mice, in the splash test, a measure of emotionality that is independent from physical performance (Santarelli et al, 2003; Surget et al, 2008). In fact, the switch from EE to SE was associated with a decrease in self-grooming, a behavior also found in chronically stressed mice (Santarelli et al, 2003; Surget et al, 2008). In the OF test, EE-SE mice explored the center of the arena less than EE-EE and SE-EE mice, suggesting that the effects of EE on this anxiety-related behavior are rapidly lost when EE is discontinued and, conversely, that they can appear rapidly when EE is implemented. However, the OF test was not able to discriminate between EE-SE and $\mathrm{SE}-\mathrm{SE}$ mice as both these groups showed low levels of exploration. Therefore, the negative emotional consequences of environmental switch from EE to SE appear to be subtle and may be revealed by some but not all behavioral tests commonly used to study stress-related responses.

An important aspect of the negative effects of deprivation of EE is that they appear to be age-dependent. In fact, only when exposure to EE occurred at early stages of life, switch from $\mathrm{EE}$ to $\mathrm{SE}$ increased the rewarding effects of cocaine. In contrast, when exposure to EE occurred at adulthood, switch from EE to SE simply lead to the loss of the effects of EE but not to an increase in the rewarding effects of cocaine. Therefore, these results support the hypothesis that adolescence and young adulthood represent critical periods of development during which external factors and environmental conditions may have a great impact on the vulnerability to drugs (Ernst et al, 2009; Marco et al, 2011; Spear, 2000). On the other hand, during adulthood, individuals may be more able to cope and overcome environmental changes even when they may represent a deterioration compared with previous conditions.

The CRF system is known to have a major role in stress responses and emotions (Arborelius et al, 1999). In addition, this system has been recently shown to be responsible for the anxiolytic effects of EE (Sztainberg et al, 2010) and in the stress-induced increases in cocaine CPP (Kreibich et al, 2009), suggesting that it may also be involved in the behavioral consequences of environmental switch. In addition, CRF has been shown to have major roles in drug-taking and drug-seeking behavior (Corominas et al, 2010; Koob, 2008), and in models of relapse (Buffalari et al, 2011; Shalev et al, 2010). In agreement with this role, we found that switch from $\mathrm{EE}$ to SE produces significant increases in CRF mRNA levels in the BNST, a brain region critically involved in stress responses and emotions (Davis and Whalen, 2001). The causal role of the CRF system, especially $C_{R F} R$, in the negative consequence of switching from $\mathrm{EE}$ to $\mathrm{SE}$ on the vulnerability to addiction was confirmed by the fact that chronic blockade of $\mathrm{CRF}_{1} \mathrm{R}$ by antalarmin prevented the enhancement of the rewarding effects of cocaine. Similarly, in a previous study, it was found that injections of antalarmin prior to exposure to a forced swim stress prevented an enhancement in cocaine CPP (Kreibich et al, 2009). In the same study, it was found that forced swim stress produced an increase in CREB phosphorylation in the NAc shell, NAc core, LS, the BNST, the BLA, and CeA, and that CREB-deficient mice did not show any stress-induced enhancement of cocaine CPP (Kreibich et al, 2009). In the present study, the switch from EE to SE increased pCREB levels in the NAc shell and in the BNST but not in the other brain areas investigated, suggesting that different stressors can induce different patterns of CREB activation. Increases in PCREB in the BNST and NAc shell have been shown to be associated with depression-like behaviors, enhanced responses to stress, and anxiety (Carlezon et al, 2005; Curtis et al, 2002; Kreibich et al, 2009). Although the role of CREB in the NAc on cocaine reward has been clearly established (Carlezon et al, 2005; Kreibich et al, 2009), future studies will be necessary to better define the role of CREB in the BNST on these effects. Nevertheless, a recent study has shown that CREB phosphorylation in the BNST is necessary for stress-induced reinstatement of cocaine CPP (Briand et al, 2010).

An important implication of our findings is that although positive life conditions, if maintained, may provide protection from drug addiction (Carroll et al, 2009; Laviola et al, 2008; Stairs and Bardo, 2009), discontinuation of EE increases vulnerability to drug addiction. Although these results deserve confirmation with operant self-administration procedures, they may have implication for the understanding of the consequences of changes in environmental conditions that may occur in the passage from adolescence to adulthood. In fact, although it is generally agreed that addiction is associated with poor socioeconomic and poor family conditions (Swadi, 1999), many people belonging to families with conditions that appear rather positive also develop addiction to drugs and in particular to cocaine. Our results suggest that changes from living conditions perceived as positive to less favorable environmental conditions could lead to negative emotional states that can favor the development of psychiatric illness such as addiction.

\section{ACKNOWLEDGEMENTS}

This work was supported by Centre National pour la Recherche Scientifique, University of Poitiers, Mission Interministérielle de la Lutte contre les Drogues et la Toxicomanie (MILDT-INSERM-INCA 2007-2009) and the Contrat de Projet Etat Region (CPER) no. 5. JN and CC are recipients of $\mathrm{PhD}$ fellowship by the French Minister of 
Research. RER was recipient of a CNRS BDI-PED fellowship. We thank Steve Goldberg and Jose Moron-Concepcion for comments on the manuscript.

\section{DISCLOSURE}

The authors declare that, except for income received from their primary employer, no financial support or compensation has been received from any individual or corporate entity over the past 3 years for research or professional service and there are no personal financial holdings that could be perceived as constituting a potential conflict of interest.

\section{REFERENCES}

Arborelius L, Owens MJ, Plotsky PM, Nemeroff CB (1999). The role of corticotropin-releasing factor in depression and anxiety disorders. J Endocrinol 160: 1-12.

Bardo MT, Klebaur JE, Valone JM, Deaton C (2001). Environmental enrichment decreases intravenous self-administration of amphetamine in female and male rats. Psychopharmacology (Berl) 155: 278-284.

Baroncelli L, Braschi C, Spolidoro M, Begenisic T, Sale A, Maffei L (2010). Nurturing brain plasticity: impact of environmental enrichment. Cell Death Differ 17: 1092-1103.

Briand LA, Vassoler FM, Pierce RC, Valentino RJ, Blendy JA (2010). Ventral tegmental afferents in stress-induced reinstatement: the role of cAMP response element-binding protein. J Neurosci 30: 16149-16159.

Buffalari DM, Baldwin CK, Feltenstein MW, See RE (2011). Corticotrophin releasing factor (CRF) induced reinstatement of cocaine seeking in male and female rats. Physiol Behav 105: 209-214.

Carlezon Jr WA, Duman RS, Nestler EJ (2005). The many faces of CREB. Trends Neurosci 28: 436-445.

Carroll ME, Anker JJ, Perry JL (2009). Modeling risk factors for nicotine and other drug abuse in the preclinical laboratory. Drug Alcohol Depend 104(Suppl 1): S70-S78.

Cirulli F, Berry A, Bonsignore LT, Capone F, D’ndrea I, Aloe L et al (2010). Early life influences on emotional reactivity: evidence that social enrichment has greater effects than handling on anxiety-like behaviors, neuroendocrine responses to stress and central BDNF levels. Neurosci Biobehav Rev 34: 808-820.

Corominas M, Roncero C, Casas M (2010). Corticotropin releasing factor and neuroplasticity in cocaine addiction. Life Sci 86: 1-9.

Curtis AL, Bello NT, Connolly KR, Valentino RJ (2002). Corticotropin-releasing factor neurones of the central nucleus of the amygdala mediate locus coeruleus activation by cardiovascular stress. J Neuroendocrinol 14: 667-682.

Davis M, Whalen PJ (2001). The amygdala: vigilance and emotion. Mol Psychiatry 6: 13-34.

El Rawas R, Thiriet N, Lardeux V, Jaber M, Solinas M (2009). Environmental enrichment decreases the rewarding but not the activating effects of heroin. Psychopharmacology (Berl) 203: 561-570.

Enoch MA (2011). The role of early life stress as a predictor for alcohol and drug dependence. Psychopharmacology 214: 17-31.

Ernst M, Romeo RD, Andersen SL (2009). Neurobiology of the development of motivated behaviors in adolescence: a window into a neural systems model. Pharmacol Biochem Behav 93: 199-211.

Goeders NE (2002). Stress and cocaine addiction. J Pharmacol Exp Ther 301: 785-789.

Green TA, Alibhai IN, Roybal CN, Winstanley CA, Theobald DE, Birnbaum SG et al (2009). Environmental Enrichment Produces a Behavioral Phenotype Mediated by Low Cyclic Adenosine Monophosphate Response Element Binding (CREB) Activity in the Nucleus Accumbens. Biol Psychiatry 67: 28-35.
Green TA, Gehrke BJ, Bardo MT (2002). Environmental enrichment decreases intravenous amphetamine self-administration in rats: dose-response functions for fixed- and progressive-ratio schedules. Psychopharmacology (Berl) 162: 373-378.

Koob GF (2008). A role for brain stress systems in addiction. Neuron 59: 11-34.

Kreek MJ, Nielsen DA, Butelman ER, LaForge KS (2005). Genetic influences on impulsivity, risk taking, stress responsivity and vulnerability to drug abuse and addiction. Nat Neurosci 8: 1450-1457.

Kreibich AS, Briand L, Cleck JN, Ecke L, Rice KC, Blendy JA (2009). Stress-induced potentiation of cocaine reward: a role for CRF R1 and CREB. Neuropsychopharmacology 34: 2609-2617.

Laviola G, Hannan AJ, Macri S, Solinas M, Jaber M (2008). Effects of enriched environment on animal models of neurodegenerative diseases and psychiatric disorders. Neurobiol Dis 31: 159-168.

Madronal N, Lopez-Aracil C, Rangel A, del Rio JA, Delgado-Garcia JM, Gruart A (2010). Effects of enriched physical and social environments on motor performance, associative learning, and hippocampal neurogenesis in mice. PLoS One 5: e11130.

Marco EM, Macri S, Laviola G (2011). Critical age windows for neurodevelopmental psychiatric disorders: evidence from animal models. Neurotox Res 19: 286-307.

Marinelli M, Piazza PV (2002). Interaction between glucocorticoid hormones, stress and psychostimulant drugs. Eur J Neurosci 16: 387-394.

McLaughlin JP, Li S, Valdez J, Chavkin TA, Chavkin C (2006). Social defeat stress-induced behavioral responses are mediated by the endogenous kappa opioid system. Neuropsychopharmacology 31: 1241-1248.

McLaughlin JP, Marton-Popovici M, Chavkin C (2003). Kappa opioid receptor antagonism and prodynorphin gene disruption block stress-induced behavioral responses. J Neurosci 23: 5674-5683.

Munn E, Bunning M, Prada S, Bohlen M, Crabbe JC, Wahlsten D (2011). Reversed light-dark cycle and cage enrichment effects on ethanol-induced deficits in motor coordination assessed in inbred mouse strains with a compact battery of refined tests. Behav Brain Res 224: 259-271.

Nithianantharajah J, Hannan AJ (2009). The neurobiology of brain and cognitive reserve: Mental and physical activity as modulators of brain disorders. Prog Neurobiol 89: 369-382.

Pietropaolo S, Feldon J, Alleva E, Cirulli F, Yee BK (2006). The role of voluntary exercise in enriched rearing: a behavioral analysis. Behav Neurosci 120: 787-803.

Rosenzweig MR, Bennett EL (1996). Psychobiology of plasticity: effects of training and experience on brain and behavior. Behav Brain Res 78: 57-65.

Santarelli L, Saxe M, Gross C, Surget A, Battaglia F, Dulawa S et al (2003). Requirement of hippocampal neurogenesis for the behavioral effects of antidepressants. Science 301: 805-809.

Shalev U, Erb S, Shaham Y (2010). Role of CRF and other neuropeptides in stress-induced reinstatement of drug seeking. Brain Res 1314: 15-28.

Sinha R (2001). How does stress increase risk of drug abuse and relapse? Psychopharmacology (Berl) 158: 343-359.

Solinas M, Chauvet C, Thiriet N, El Rawas R, Jaber M (2008). Reversal of cocaine addiction by environmental enrichment. Proc Natl Acad Sci USA 105: 17145-17150.

Solinas M, Thiriet N, Chauvet C, Jaber M (2010). Prevention and treatment of drug addiction by environmental enrichment. Prog Neurobiol 92: 572-592.

Solinas M, Thiriet N, El Rawas R, Lardeux V, Jaber M (2009). Environmental enrichment during early stages of life reduces the behavioral, neurochemical, and molecular effects of cocaine. Neuropsychopharmacology 34: 1102-1111. 


\section{Enrichment deprivation and cocaine reward}

J Nader et al

Spear LP (2000). The adolescent brain and age-related behavioral manifestations. Neurosci Biobehav Rev 24: 417-463.

Stairs DJ, Bardo MT (2009). Neurobehavioral effects of environmental enrichment and drug abuse vulnerability. Pharmacol Biochem Behav 92: 377-382.

Stairs DJ, Klein ED, Bardo MT (2006). Effects of environmental enrichment on extinction and reinstatement of amphetamine self-administration and sucrose-maintained responding. Behav Pharmacol 17: 597-604.

Stern CM, Luoma JI, Meitzen J, Mermelstein PG (2011). Corticotropin releasing factor-induced CREB activation in striatal neurons occurs via a novel Gbetagamma signaling pathway. PLoS One 6: e18114.

Surget A, Saxe M, Leman S, Ibarguen-Vargas Y, Chalon S, Griebel $\mathrm{G}$ et al (2008). Drug-dependent requirement of hippocampal neurogenesis in a model of depression and of antidepressant reversal. Biol Psychiatry 64: 293-301.

Swadi H (1999). Individual risk factors for adolescent substance use. Drug Alcohol Depend 55: 209-224.
Sztainberg Y, Kuperman Y, Tsoory M, Lebow M, Chen A (2010). The anxiolytic effect of environmental enrichment is mediated via amygdalar CRF receptor type 1. Mol Psychiatry 15: 905-917.

Thiriet N, Amar L, Toussay X, Lardeux V, Ladenheim B, Becker KG et al (2008). Environmental enrichment during adolescence regulates gene expression in the striatum of mice. Brain Res 1222: 31-41.

Thiriet N, Gennequin B, Lardeux V, Chauvet C, Decressac M, Janet $\mathrm{T}$ et al (2011). Environmental enrichment does not reduce the rewarding and neurotoxic effects of methamphetamine. Neurotox Res 19: 172-182.

van der Veen R, Piazza PV, Deroche-Gamonet V (2007). Geneenvironment interactions in vulnerability to cocaine intravenous self-administration: a brief social experience affects intake in DBA/ 2J but not in C57BL/6J mice. Psychopharmacology 193: 179-186.

Xu Z, Hou B, Gao Y, He F, Zhang C (2007). Effects of enriched environment on morphine-induced reward in mice. Exp Neurol 204: 714-719.

Supplementary Information accompanies the paper on the Neuropsychopharmacology website (http://www.nature.com/npp) 